\title{
Discovery of novel astrovirus genotype species in small ruminants
}

\author{
Ronja V Kauer ${ }^{\text {Equal first author, } 1}$, Michel C Koch ${ }^{\text {Equal first author, } 1,2}{ }^{\text {, Melanie M Hierweger }}{ }^{1,2}$, Simea Werder ${ }^{1}$, Céline L Boujon ${ }^{1,2}$ \\ , Torsten Seuberlich ${ }^{\text {Corresp. } 1}$ \\ ${ }^{1}$ Division of Neurological Sciences, Vetsuisse Faculty, University of Bern, Bern, Switzerland \\ 2 Graduate School for Cellular and Biomedical Sciences, University of Bern, Bern, Switzerland \\ Corresponding Author: Torsten Seuberlich \\ Email address: torsten.seuberlich@vetsuisse.unibe.ch
}

Astroviruses are single-stranded, positive-sense RNA viruses, best known for causing diarrhea in humans and are also found in many other mammals; in those, the relevance in gastroenteritis remains unclear. Recently described neurotropic astroviruses showed associations with encephalitis in humans as well as in other mammals. In Switzerland, two different neurotropic astroviruses were identified in cattle, as well as one in a sheep. The high genetic similarity between the ovine and one of the bovine astroviruses strengthens the hypothesis of an interspecies transmission. In humans, astroviruses associated with encephalitis were found also in human stool samples, suggesting that in these patients the infection spreads from the gastrointestinal tract to the brain under certain conditions, such as immunosuppression. Whether a similar pathogenesis occurs in ruminants remains unknown. The aims of this study were 1) the investigation of the potential occurrence of neurotropic astroviruses in feces samples, 2) the discovery and analysis of so far unknown astroviruses in small ruminants and other ruminant species' fecal samples and 3) the examination of a potential interspecies transmission of astroviruses. To achieve the aims, RNA extraction out of 164 fecal samples from different ruminant species was performed and all samples were screened for known neurotropic astroviruses occurring in Switzerland, as well as for various astroviruses using RT-PCR. Positive tested samples were submitted to next generation sequencing (NGS). The generated sequences were compared to nucleotide- and amino acid databases, virus properties were identified, and phylogenetic analyses as well as recombination analysis were performed. The excretion of neurotropic astroviruses in small ruminants' feces could not be demonstrated, but this work suggests the first identification of astroviruses in goats as well as the discovery of multiple and highly diverse new genetic variants in small ruminants, which lead to a classification into novel genotype-species. Additionally, the prediction of multiple recombination events in four of five newly discovered full or almost full-length genome 
sequences suggests a plausible interspecies transmission. The findings point out the occurrence and fecal shedding of previously unknown astroviruses in sheep and goats and pave the way towards a better understanding of the diversity and transmission of astroviruses in small ruminants. 
1 Discovery of novel astrovirus genotype species in small

2

3

4 Ronja Véronique Kauer ${ }^{1}$, Michel Christoph Koch $^{1,2}$, Melanie Michaela Hierweger ${ }^{1,2}$, Simea

5

6

$7 \quad{ }^{1}$ Division of Neurological Sciences, Vetsuisse Faculty, University of Bern, Bern, Switzerland

$8{ }^{2}$ Graduate School for Cellular and Biomedical Sciences, University of Bern, Bern, Switzerland

Prof. Dr. Torsten Seuberlich ${ }^{\#}$

Division of Neurological Sciences, Vetsuisse Faculty, University of Bern, Bremgartenstrasse

109a, CH-3012 Bern, Switzerland

13

Email: torsten.seuberlich $@$,vetsuisse.unibe.ch 


\section{Abstract}

Astroviruses are single-stranded, positive-sense RNA viruses, best known for causing diarrhea in humans and are also found in many other mammals; in those, the relevance in gastroenteritis remains unclear. Recently described neurotropic astroviruses showed associations with encephalitis in humans as well as in other mammals. In Switzerland, two different neurotropic astroviruses were identified in cattle, as well as one in a sheep. The high genetic similarity between the ovine and one of the bovine astroviruses strengthens the hypothesis of an interspecies transmission. In humans, astroviruses associated with encephalitis were found also in human stool samples, suggesting that in these patients the infection spreads from the gastrointestinal tract to the brain under certain conditions, such as immunosuppression. Whether a similar pathogenesis occurs in ruminants remains unknown. The aims of this study were 1) the investigation of the potential occurrence of neurotropic astroviruses in feces samples, 2) the discovery and analysis of so far unknown astroviruses in small ruminants and other ruminant species' fecal samples and 3) the examination of a potential interspecies transmission of astroviruses.

To achieve the aims, RNA extraction out of 164 fecal samples from different ruminant species was performed and all samples were screened for known neurotropic astroviruses occurring in Switzerland, as well as for various astroviruses using RT-PCR. Positive tested samples were submitted to next generation sequencing (NGS). The generated sequences were compared to nucleotide- and amino acid databases, virus properties were identified, and phylogenetic analyses as well as recombination analysis were performed.

The excretion of neurotropic astroviruses in small ruminants' feces could not be demonstrated, but this work suggests the first identification of astroviruses in goats as well as the discovery of 
multiple and highly diverse new genetic variants in small ruminants, which lead to a classification into novel genotype-species. Additionally, the prediction of multiple recombination events in four of five newly discovered full or almost full-length genome sequences suggests a plausible interspecies transmission. The findings point out the occurrence and fecal shedding of previously unknown astroviruses in sheep and goats and pave the way towards a better understanding of the diversity and transmission of astroviruses in small ruminants.

\section{Introduction}

Astroviruses (AstV) are non-enveloped, single-stranded positive-sense RNA viruses with an icosahedral virion structure, appearing as a star-like shape in electron microscopy (Caul \& Appleton 1982). The AstV genome is $6.2-7.8 \mathrm{~kb}$ in size and polyadenylated at the 3 ' end. It presents at least three open reading frames (ORF): ORF1a, ORF1ab and ORF2. ORF1a and ORF1ab encode nonstructural precursor proteins, nspla and nsplab. The latter is translated via a ribosomal frameshift mechanism, where ORF1b is translated together with ORF1a (Marczinke et al. 1994). ORF2 encodes the capsid precursor protein, which is then intra- and extracellularly further processed to mature structural proteins (Willcocks et al. 1994).

According to the affected host class, two astrovirus genera were established: Mamastroviruses (MAstV) representing genotype species affecting mammalian species and Avastroviruses (AAstV) containing those viruses found in avian species. Due to the availability of high throughput next-generation-sequencing (NGS) technologies and the use of broadly reactive panastrovirus RT-PCR protocols, there has been a remarkable increase in the number of astroviruses discovered in diverse species during the last years (Boujon et al. 2017a). Astroviruses were first described in 1975 in a human stool sample (Appleton \& Higgins 1975; Madeley \& Cosgrove 1975). In humans, AstV are best known as a major source of outbreaks of gastroenteritis, 
61 especially in infants, young children and immunocompromised people (De Benedictis et al.

62 2011; Fischer et al. 2017). However, intestinal tissue infected with AstV shows only minor

63 histological changes such as a mild intestinal inflammatory response (Sebire et al. 2004) and the

64 knowledge on the pathogenesis of gastroenteric disease associated with astroviruses is still

65 limited (Moser \& Schultz-Cherry 2005).

66 In 2010, astroviruses were found for the first time in association with encephalitis in a child with

67 immunodeficiency (Quan et al. 2010). Thereafter, several novel AstV genotype species were

68 detected in other human encephalitis cases (Brown et al. 2015; Lum et al. 2016). Encephalitis-

69 associated AstV could be detected in stool samples, as well as in other body fluids, such as

70 cerebrospinal fluid and plasma, suggesting that in these patients the infection spreads from the

71 gastrointestinal tract to the brain (Cordey et al. 2016).

72 In animals, the state of knowledge about the tissue tropism of AstV is even more limited. Even

73 though the presence of ovine astroviruses (OvAstV) in fecal sheep samples constituted the first

74 report of astroviruses in animals (Snodgrass \& Gray 1977), still little is known about astrovirus

75 infections in small ruminants, their transmission within and across species as well as their

76 association with disease. In recent years a wide variety of mammalian domestic animal species

77 were found positive for astroviruses in their feces; e.g. cattle (Woode \& Bridger 1978), sheep

78 (Snodgrass \& Gray 1977), red deer (Tzipori et al. 1981), takins (Guan et al. 2018) and also

79 domestic carnivores (Hoshino et al. 1981; Williams 1980), mice (Kjeldsberg \& Hem 1985) and

80 pigs (Bridger 1980), but their role in the context of disease remained largely unclear.

81 Remarkably, almost at the same time as the discovery of the first astrovirus-associated

82 encephalitis in humans, the so-called shaking mink syndrome was described, which could be

83 traced back neurovirulent astrovirus infection (Blomstrom et al. 2010). One year later, in 2011, a 
84 neurovirulent porcine astroviruse type 3 could be identified as the cause of disease in outbreaks

85 of meningoencephalomyelitis in piglets (Laurin et al. 2011; Reuter et al. 2012).

86 Since 2013, different novel AstV genotype species were found as a plausible cause of non-

87 suppurative encephalitis in cattle (Bouzalas et al. 2014; Li et al. 2013; Schlottau et al. 2016) and

88 a few years later also in sheep (Pfaff et al. 2017). In Switzerland, three neurotropic astroviruses

89 were identified in brain-tissue of ruminants; bovine astrovirus $\mathrm{CH} 13$ (BoAstV-CH13) and bovine

90 astrovirus CH15 (BoAstV-CH15) in cattle (Bouzalas et al. 2014; Seuberlich et al. 2016), as well

91 as ovine astrovirus $\mathrm{CH} 16$ (OvAstV-CH16) in sheep. The capsid protein as well as the non-

92 structural proteins of this encephalitis-associated astrovirus in sheep (OvAstV-CH16) show a

93 high similarity - around $99 \%$ on both the nucleotide and the amino acid level - to bovine

94 astrovirus $\mathrm{CH} 15$, suggesting interspecies transmission of this genotype species between sheep

95 and cattle (Boujon et al. 2017b).

96 To date, there are 19 genotype species of Mamastrovirus (MAstV 1-19) recognized by the

97 International Committee on Taxonomy of Viruses (ICTV). In particular, in ovine astroviruses,

98 little is known about their diversity. Ovine astrovirus 1 belongs to Mamastrovirus 13 and is the

99 only enterotropic astrovirus closely related to neurotropic strains, but their exact taxonomy is still

100 pending (Boujon et al. 2017a). Based on phylogenetic analyses of different viral strains of

101 bovine, ovine and porcine origin, further evidence of possible interspecies transmission could be

102 found (Donato \& Vijaykrishna 2017). The close clustering of farmed animals' astrovirus strains

103 reinforce the assumption of probable interspecies transmission events.

104 The aims of the present study included the assessing of a potential shedding of neurotropic

105 astroviruses in fecal samples, the investigation of diverse astroviruses in different ruminant

106 species and the examination of a potential interspecies transmission. Fecal samples of sheep, 
107 goats, deer, alpaca and llamas were tested for BoAstV-CH13 as well as BoAstV-CH15/OvAstV-

$108 \mathrm{CH} 16$ and screened for other astroviruses using a pan-astrovirus RT-PCR. NGS and

109 bioinformatics were used to recover viral genome sequences and to perform a phylogenetic

110 comparison as well as a recombination analysis with other known astroviruses.

\section{Materials and Methods}

\section{Samples}

113 Fecal samples submitted for parasitological diagnostics were kindly donated by the Institute of

114 Parasitology, Vetsuisse Faculty, University of Bern (Bern, Switzerland). In total 164 fecal

115 samples, derived from 56 sheep, 56 goats, 30 alpacas, 12 deer, 4 llamas, 2 bisons, 2 chamois, 1

116 giraffe and 1 ibex were investigated. Additional information on the animals and their health

117 status was not available. Fecal samples were suspended 1:10 (w/v) in sterile PBS (137 mM

$118 \mathrm{NaCl}, 10 \mathrm{mM}$ Phosphate, $2.7 \mathrm{mM} \mathrm{KCl}$; $\mathrm{pH}$ 7.4) and stored at $-80^{\circ} \mathrm{C}$ until further analysis.

119 BoAstV-CH13 and OvAstV-CH16 positive and negative brain tissues were used as positive and 120 negative controls for RT-PCRs and were available from the archive of the Division of

121 Neurological Sciences, Vetsuisse Faculty, University of Bern (Bern, Switzerland). The summary

122 of the laboratory workflow is shown in Figure 1.

123

124

125

126

127

128

129

\section{RNA Extraction}

All RNA extractions from fecal suspensions for AstV-screening by RT-PCR were done with the QIAamp Viral RNA Mini Kit (Qiagen) and RNA isolation from brain tissue was performed with TRI Reagent (Sigma-Aldrich) according to the manufacturers' protocols. To prepare samples for NGS, $500 \mu \mathrm{L}$ of feces-suspensions were centrifuged at 16'000 x g for 3min. The supernatants were then centrifuged through Vivaclear MINI Clarifying filters with $0.8 \mu \mathrm{m}$ PES (Sartorius) at $2.000 \mathrm{x}$ g for $5 \mathrm{~min}$. Next, $280 \mu \mathrm{L}$ of the filtrates were treated with $2 \mu \mathrm{L}$ Benzonase $(1 \mathrm{U} / \mu \mathrm{L})$ 
130 (Merck) for 2 hours at $37^{\circ} \mathrm{C}$. The benzonase was inactivated by adding EDTA to a final

131 concentration of 5mM. Finally, RNA extraction was performed with the QIAamp Viral RNA

132 Mini Kit (Qiagen) with the modification that the carrier RNA was omitted. RNA extracts were

133 stored at $-80^{\circ} \mathrm{C}$ until further analysis.

134

135

136

137

138

139

140

141

142

143

144

145

146

147

148

149

150

151

152

153

\section{Detection of neurotropic astroviruses BoAstV-CH13 and BoAstV-CH15/OvAstV- CH16 by RT-qPCR}

To detect BoAstV-CH13, the RNA extracts were analyzed by either of two different probe based

RT-qPCR assays (CH13-A or CH13-B) as described previously. While the CH13-A assay targets

the 5' part of the viral genome in ORF1a, the CH13-B assay detects the center of the viral

genome in ORF2. Both protocols revealed a very similar excellent accuracy, precision, and

analytical sensitivity (Lüthi et al. 2018).

For the detection of BoAstV-CH15 as well as OvAstV-CH16 a primer pair and a probe were designed based on full-genome sequence alignments of the following three strains from Switzerland and Germany; BoAstV-CH15, OvAstV-CH16 and BoAstV-BH89/14, targeting the 3' end of ORF2 using the Geneious software package (Biomatters, version 11.1.4). The RTqPCR was performed as described previously (Kuchler et al. 2019). After each cycle, the fluorescence was measured with the FAM channel and data was analyzed using the Sequence Detection Software (Applied Biosystems, Version 1.4) with automatic baseline detection and a manual threshold of 0.2 . The positive-negative cut-off was set at the cycle threshold (ct) value of 35. Each RT-qPCR run was performed using a positive and negative brain tissue control as well as a water control. All primers and probes are provided in Supplementary Table 1.

\section{Pan-astrovirus RT-PCR}

For the detection of other astroviruses, a previously described heminested RT-PCR protocol using five degenerated primers; four forward and one reverse primer, which target a 450nt long 
154 sequence at the 3'end of ORF 1b of a broad panel of astroviruses was used (Chu et al. 2008).

155 Primer sequences are provided in Supplementary Table 1. First-Strand cDNA synthesis was

156 performed using the GoScript reverse transcriptase (Promega) and the gene specific reverse

157 primer. The heminested PCR was done with a GoTaq Green Master Mix System (Promega) in

158 two reaction rounds. For the first round, the PCR was set up in $25 \mu \mathrm{L}$ reactions containing

$15912,5 \mu \mathrm{L}$ of $2 \mathrm{x}$ GoTaq Green Master Mix, 4,5 $\mu \mathrm{L}$ of cDNA and a mixture of two forward primers

160 PanAstV forward 1 and -2 and the PanAstV reverse primer, each in final concentration of

$16110 \mu \mathrm{M}$ (Chu et al. 2008). The PCR was carried out with the following setting: $2 \mathrm{~min}, 95^{\circ} \mathrm{C}$ and 30

162 cycles each of $30 \mathrm{~s}, 95^{\circ} \mathrm{C} ; 30 \mathrm{~s}, 50^{\circ} \mathrm{C} ; 30 \mathrm{~s}, 72^{\circ} \mathrm{C}$ and final elongation $7 \mathrm{~min}, 72^{\circ} \mathrm{C}$. For the second

163 round, the same reverse primer PanAstV-reverse was used as well as a mixture of

164 PanAstV_forward_nested 1 and -2 (final concentration of $10 \mu \mathrm{M}$ ) and $1 \mu \mathrm{L}$ of the first-round

165 PCR-product as a template. Temperature settings were the same as in the first PCR, but with 40

166 cycles instead of 30 cycles.

\section{Next Generation Sequencing}

168 Prior to Next Generation Sequencing (NGS), libraries were prepared using TruSeq DNA Nano

169 Kit (Illumina). For cDNA synthesis and polyA-selection the SMARTer Ultra Low Input RNA

170 Kit (Takara Bio Inc.) was applied. NGS was performed on an Illumina HiSeq 3000 or NovaSeq

1716000 in paired-end mode (2x150bp).

\section{Bioinformatics analysis}

173 Reads were quality-trimmed with trimmomatic (Ver. 0.36) and mapped to their respective host

174 genomes (alpaca: BioProject PRJNA30567, assembly Vicugna_pacos-2.0.1, deer: BioProject

175 PRJNA324173, assembly CerEla1.0, goat: BioProject PRJNA340281, assembly ARS1, sheep: 
176 BioProject PRJNA179263, assembly Oar_v4.0) using STAR (Ver. 2.5.3a). Quality-trimmed and

177 unmapped reads were assembled via SPAdes (Ver. 3.11.1).

178 Resulting scaffolds were then aligned to virus databases (Genbank and RefSeq viral nucleotide

179 sequences downloaded on $12^{\text {th }}$ of April 2018, UniProt viral amino acid sequences downloaded

180 on $12^{\text {th }}$ of March 2018) using BLASTn (Ver. 2.7.1+, default settings) and DIAMOND (Ver.

181 0.9.10, default settings). In order to exclude false positives, the scaffolds with a virus hit were

182 aligned to an in-house non-viral database consisting of archaeal, bacterial, fungal, mammal and

183 protozoal sequences. Scaffolds were considered false positive if they had a longer hit on a

184 sequence of the in-house database compared to the virus databases or if they had a nucleotide hit

185 of more than $10 \%$ of their own length to any sequence of the non-viral database. Scaffolds with

186 hits to astroviruses on nucleotide and/or amino acid level were considered valid astrovirus hits.

187 For further analysis, scaffolds with a length over 5'550 nt and the three astrovirus-typical open

188 reading frames including the ribosomal frameshift sequence between ORF1a and ORF1b were

189 selected.

\section{Rapid amplification of cDNA ends}

191 In order to complete the inchoate 5 ' ends of the five almost full-length scaffolds, a rapid

192 amplification of cDNA ends (RACE) was performed. This 5' RACE was carried out with a

193 RACE-System (Invitrogen ${ }^{\mathrm{TM}}$ ) according to the manufacturer's instructions except of the usage of

194 SuperScript ${ }^{\mathrm{TM}}$ III Reverse Transcriptase (Invitrogen ${ }^{\mathrm{TM}}$ ) instead of SuperScript ${ }^{\mathrm{TM}}$ II Reverse

195 Transcriptase (Invitrogen ${ }^{\mathrm{TM}}$ ). Nested PCR was performed with gene specific primers

196 (Supplementary Table 1) and Taq DNA Polymerase with Standard Taq Buffer (New England

197 BioLabs, Inc.) as described by the manufacturer and an annealing temperature of $55^{\circ} \mathrm{C}$. 
198 After visualization on a 1\% agarose gel, PCR-products were excised of the gel and purified using

199 NucleoSpin Gel and PCR Clean-up system (Macherey-Nagel) according to the manufacturer's 200 protocol.

201 Each $3 \mu 1$ of the purified PCR products was Sanger-sequenced using the BigDye ${ }^{\circledR}$ Terminator

202 v3.1 Cycle Sequencing Kit (Applied Biosystems) in a 3730 DNA Analyzer (Applied

203 Biosystems) according to the manufacturer's protocol. Sequence data were analyzed using the

204 Geneious software package (Version 10.2.6), trimmed with an error probability limit of 0.05 on

205 both ends and aligned to the respective scaffolds.

206 Phylogenetic analysis

207 Capsid precursor sequences of 36 astrovirus strains were used for phylogenetic analysis and

208 included astrovirus strains of various ruminants and our newly discovered sequences. Based on

209 these sequences, a phylogenetic tree was constructed using the Maximum Likelihood method

210 with 1000 bootstrap replicates based on the Le_Gascuel_2008 with Freqs. model (Le \& Gascuel

211 2008) in MEGA 7 (Ver. 7.0.26). The model was chosen using the Find Best DNA/Protein

212 Models option in MEGA 7.

\section{Recombination analysis}

214 Putative recombination events were assessed using the Recombination Detection Program

215 (RDP4, Ver. 4.94) (Martin et al. 2015), following the RDP4 Instruction Manual

216 (http://web.cbio.uct.ac.za/ darren/RDP4Manual.pdf) and with the highest acceptable p-value set

217 to 0.01 . Recombination events were considered only when involving at least one of our newly

218 generated sequences and having a highest acceptable p-value of 0.01 with all of the following

219 methods: RDP (Martin \& Rybicki 2000), GENECONV (Padidam et al. 1999), Bootscan (Martin 
220 et al. 2005), Maxchi (Smith 1992), Chimaera (Posada \& Crandall 2001), SiScan (Gibbs et al.

221 2000) and 3Seq (Lam et al. 2018).

\section{Confirmation of newly discovered astrovirus sequences}

223 To confirm novel astrovirus sequences, discovered by NGS, a RT-qPCR using specific primer-

224 probe-combinations for each putative virus-candidate was applied. Specific primers and probes

225 were designed for each of the almost full-length scaffolds using the Geneious software package

226 (Biomatters, version 11.1.4). The primer and probe sequences with the respective nucleotide

227 positions of the target are provided in Supplementary Table 1.

228 All RT-qPCR reactions were performed using the TagMan ${ }^{\mathrm{TM}}$ Fast Virus 1-Step Master Mix

229 (Applied Biosystems) in $10 \mu \mathrm{L}$ reactions according to the manufacture's inctructions with a final

230 concentration of $500 \mathrm{nM}$ for each primer and $125 \mathrm{nM}$ for each probe. For each reaction $2 \mu \mathrm{L}$

231 RNA were added to $8 \mu \mathrm{L}$ Master-Mix. The RT-qPCR was performed using a CFX96 ${ }^{\mathrm{TM}}$ Real

232 Time System on C1000 Touch ${ }^{\mathrm{TM}}$ Thermal Cycler (BioRad). With the following cycle settings:

$23310 \mathrm{~min}, 45^{\circ} \mathrm{C} ; 10 \mathrm{~min}, 95^{\circ} \mathrm{C}$ and 40 cycles $\left(15 \mathrm{sec}, 95^{\circ} \mathrm{C} ; 20 \mathrm{sec}, 61^{\circ} \mathrm{C} ; 30 \mathrm{sec}, 60^{\circ} \mathrm{C}\right)$. After each

234 elongation step, fluorescence was measured and analyzed with the CFX Maestro software

235 (BioRad, version 4.1.2433.1219) with an auto calculated baseline threshold. Samples with a ct-

236 value $<35$ were defined as positive.

237

238

239

240

241

\section{Retrospective screening of samples for newly discovered astrovirus-candidates}

After confirmation of all newly discovered astrovirus-candidates, all remaining 159 samples

were re-tested using the RT-qPCR as described above. For each run, a non-template control was used as negative control. The respective confirmed samples served as target-specific positive controls. 


\section{Results}

243 Detection of neurotropic astroviruses BoAstV-CH13 and OvAstV-CH16 by RT244 qPCR

245 Quantitative RT-PCRs were performed for the detection of neurotropic astroviruses. Positive controls showed ct-values on average of 22.73 (22.62-22.86) for BoAstV-CH13 and 23.22

247 (21.74-24.61) for OvAstV-CH16, respectively. All negative controls were scored negative. All

248164 fecal samples had ct-values $>35$ or were reported as "undetectable" and were therefore 249 diagnosed as negative for BoAstV-CH13 and OvAstV-CH16.

250

251

252

253

254

255

256

257

258

259

260

261

262

263

264

\section{Detection of various astroviruses using Pan-astrovirus RT-PCR}

To ensure the effective implementation and to establish positive controls, the protocol was first applied to brain-extracts of BoAstV-CH13 and OvAstV-CH16 astrovirus-positive animals. These samples showed a strong and clear band with the expected size of $450 \mathrm{bp}$. Nineteen of the 164 tested fecal samples derived from sheep ( $\mathrm{n}=6$ : $\mathrm{S} 1$ - S6), goats $(\mathrm{n}=8$ : $\mathrm{G} 1$ - G8), alpaca $(\mathrm{n}=3$ : A1, A3, A4) and deer ( $n=2$ : D2, D5) showed a band at 450 bp and were therefore defined as Panastrovirus RT-PCR-positive.

All nineteen samples, which were positive tested in Pan-astrovirus RT-PCR were further processed for NGS. Therefore, RNA was de novo extracted, capsid-bound viral genomes were relatively enriched and free nucleic acids were depleted using benzonase treatment.

\section{NGS and bioinformatics analysis}

Sequencing in paired-end mode (2x150bp) generated $45^{\prime} 455^{\prime} 879$ to $233^{\prime} 701^{\prime} 511$ reads per sample and raw reads were deposited in the European Nucleotide Archive (Accessions ERR3143214 - ERR3143223). In 12 samples, a total of 29 scaffolds ranging from 521 to 6255 bp in size, showed astrovirus hits in the bioinformatics analysis on amino acid level, with 
265 sequence identity to known astrovirus sequences ranging from 39.3\% to 96.4\% (Supplementary

266 Table 2). The vast majority of these hits was to astrovirus strains identified in bovine fecal

267 samples. Strikingly, in one sample of a sheep (sample S3), a $967 \mathrm{nt}$ scaffold had a hit on the

268 neurotropic bovine astrovirus CH13, isolate 42799 (Bouzalas et al. 2016) on nucleotide (identity

$26983.4 \%$, hit length 296 nucleotides) as well as on amino acid (identity 57.2\%, hit length 306

270 amino acids) level. The k-mer coverage of this scaffold was $<\mathrm{x} 4$, which indicates relatively low

271 RNA concentrations. The sequence mapped to the 3' half of ORF2, which encodes the

272 hypervariable part of the capsid precursor protein (Babkin et al. 2012).

273 For the remaining astrovirus hits, we further analyzed scaffolds with a minimum length of

274 5500nt and covering parts of all three astrovirus-typical ORFs. One such scaffold was identified

275 in two sheep and in three goats (Supplementary Table 2). These tentative astrovirus scaffolds

276 were designated CapAstV-G2.1, -G3.1 and -G5.1 and OvAstV-S5.1 and -S6.1. Each of these

277 scaffolds showed the ribosomal slippery sequence (5'-AAAAAAC-3') at the ORF1a/1b junction

278 and an overlap between ORF1b and ORF2. The translated nsp1ab sequences had an identity

279 between $71.7 \%$ and $75.4 \%$ to the next best hit while the capsid protein precursor ranged from

$28057.5 \%$ to $73.7 \%$ (Table 1). Best hits were astrovirus strains previously described in cattle (Nagai

281 et al. 2015; Tse et al. 2011), deer (Smits et al. 2010) and takin (Guan et al. 2018). Taken

282 together, these findings clearly support the notion that these five scaffolds represent complete or 283 almost complete viral genomes of novel MAstV strains.

\section{Rapid amplification of cDNA ends}

285 In four of the five samples analyzed, the 5' RACE did work and Sanger sequencing added to the

2865 ' end of the respective almost full Astrovirus scaffolds (G3.1, G5.1, S5.1, S6.1). This resulted in

287 the completion of these four scaffolds, which, together with CapAstV-G2.1, were deposited on 
288 GenBank (Accessions: CapAstV-G2.1 [MK404645.1], CapAstV-G3.1 [MK404646.1],

289 CapAstV-G5.1 [MK404647.1], OvAstV-S5.1 [MK404648.1] and OvAstV-S6.1 [MK404649.1]).

\section{Phylogenetic analysis}

291 To assess the genetic relationship of the newly discovered strains and partial sequences to other

292 known MAstV, a phylogenetic comparison of all generated putative genomes to representative

293 MAstV genotype species and so far unclassified strains with high sequence similarity based on

294 the capsid precursor protein sequences (Figure 2) was conducted. For the goats, all three novel

295 CapAstV strains clustered in different branches of the phylogenetic tree. CapAstV-G2.1 and

296 CapAstV-G3.1 are both related to two different BoAstV strains: BoAstV/JPN/Hokkaido 11-

297 55/2009 (p-dist. 0.293) and BoAstV-B170/HK (p-dist. 0.361), respectively. CapAstV-G5.1 is

298 very similar to the new OvAstV strains S5.1 (p-dist. 0.236) and S6.1 (p-dist. 0.027), clustering

299 together in one clade of the phylogenetic tree. They cluster with a p-distance of 0.419 to the

300 closest related astrovirus strain CcAstV-1/DNK/2010, which has been detected in feces from

301 deer (Smits et al. 2010) (Table 1 and Figure 2). Partial sequences generated from goat feces

302 cluster together with the here described CapAstV/OvAstV or with enterotropic strains from other

303 ruminants. In the partial sequences generated from sheep and deer feces, the situation is similar

304 to the one described in goats. As previously indicated, one partial sequence, S3.1, is not

305 clustering close to other enterotropic astroviruses but rather near the neurotropic BoAstV-

$306 \mathrm{CH13/NeuroS1}$ cluster. In samples with multiple astrovirus hits, partial sequences cluster at

307 different positions of the phylogenetic tree. OvAstV-S5.1 clusters together with CapAstV-

308 G5.1/OvAstV-S6.1 and S4.1, while scaffold S5.2, detected in the same sample, clusters together

309 with sequences from sample G8 (G8.3/G8.5). 
310 Recombination analysis

311 Knowing about the high tendency toward recombination in RNA viruses (Matsui et al. 1998) and

312 previous reported indications of interspecies transmission of astroviruses based on recombination

313 analysis (De Battisti et al. 2012; Lan et al. 2011), it was decided to determine whether such

314 recombination events may have occured in the newly discovered strains. Subsequent to the

315 phylogenetic analysis, the five new astrovirus sequences were analyzed together with selected

316 astrovirus strains for plausible recombination events. Three putative recombination events were

317 reported (Supplementary Table 4). Between the three strains OvAstV-S6.1, CapAstV-G5.1 and

318 OvAstV-S5.1, two recombination events were reported, one starting at position 4062 and ending

319 at position 6114 (recombination event 1) and the other starting at position 1737 and ending at

320 position 2443 (recombination event 3) (Figure 3A) in OvAstV-S6.1. All three sequences may be

321 the recombinant. Recombination event 3 was predicted to the overlap between ORF1a and

322 ORF1b, whereas recombination event 1 was predicted for almost the entire ORF2. Another

323 putative recombination event (2) was identified between BoAstV-GX27/CHN/2014 and

324 BoAstV/JPN/Hokkaido11-55/2009 or closely related sequences as potential parental sequences,

325 with the $5^{\prime}$ ' breakpoint at position 36 and the 3 ' breakpoint at position 1154 resulting in

326 CapAstV-G2.1 as the recombinant (Figure 3B). Identities on nucleotide and amino acid level of

327 the strains involved in the recombination events can be found in Supplementary Table 3.

328

329

330

331

332

\section{Confirmation of newly discovered astrovirus sequences}

Using specific RT-qPCRs, all five newly discovered astrovirus-candidates could be confirmed with the following ct-values: G2, 27.65; G3, 25.26; G5, 26.99; S5, 25.77; S6, 25.28. The nontemplate control remained negative for all targets. Sample G5 showed a positive signal (ct-value 27.09) for the primer-probe-pair S6.1, which is not surprising, considering the fact that these two 
333 samples share highly similar sequences. In all other samples, no cross-reactivity could be

334 detected between the individual targets.

335

336

337

338

339

340

341

342

343

344

345

346

347

\section{Retrospective screening of samples for newly discovered astrovirus-candidates} All 159 remained negative for the primer-probe combinations G3.1, G5.1, S5.1 and S6.1.

Surprisingly one sample originating from a goat, scored positive for G2.1 (ct-value 29). Based on information from the original submission site (Parasitology, Vetsuisse Bern), it could be found that this sample originated from the same holding as the sample G2.

\section{Discussion}

This study presents the discovery and molecular characterization of putatively new astrovirus genotype species in sheep as well as in goats. These novel astroviruses show a broad genetic diversity compared to astroviruses affecting other species. The present results support interspecies transmission of astroviruses between goats and sheep, as well as recombination events between astroviruses affecting sheep, goats and cattle.

The aims of the study were to investigate the potential shedding of neurotropic astroviruses in small ruminants' feces, to discover and to analyze unknown astroviruses, and examine a putative interspecies transmission. Therefore, all available samples first were screened for the neurotropic astroviruses BoAstV-CH13 as well as OvAstV-CH16. Three different methods were then applied, first, highly specific RT-qPCR, second, a wide-spectrum RT-PCR, and third, NextGeneration-Sequencing of all samples interpreted as positive after RT-PCR. None of these samples could be defined as positive for BoAstV-CH13 or OvAstV-CH16. Thus, there is no evidence that neurotropic astroviruses are part of the intestinal virome of small ruminants. Noteworthy, limitations of this study are the relative small number of samples investigated, the unknown disease status of the animals and the focus mainly on sheep and goats due to a lack of 
356 available samples from other ruminant species. A related study targeting feces and brain of cattle

357 was reported previously (Oem \& An 2014).

358 Screening for various and potentially unknown astroviruses was done with a heminested RT-

359 PCR that targets a conserved sequence at the 3'end of ORF1b. However, RNA viruses, and in

360 particular astroviruses, are predicted to undergo $3.4 \times 10^{-3}$ mutations per nucleotide per genomic

361 replication (Duffy et al. 2008) and are genetically relatively diverse. Therefore, this RT-PCR

362 uses highly degenerated primers, which has the limitations that (i) it may lack sensitivity; due to

363 absence or inefficiency of primer binding to divergent astrovirus sequences and that (ii)

364 unspecific primer binding may result in amplification of non-astrovirus sequences. On the one

365 hand, as only RT-PCR positive samples were submitted to NGS, fecal samples containing

366 divergent astrovirus strains may have been classified as false negative and therefore might have

367 been excluded from further analysis. On the other hand, false positive samples may have been

368 further analyzed by NGS. Indeed, seven RT-PCR positive samples did not reveal any astrovirus

369 hit in the bioinformatics pipeline. This discrepancy may also be related to the sample

370 pretreatment procedure prior to NGS-submission.

371 Beside the discovery and investigation of four full-length and one almost full-length astrovirus

372 sequence, NGS and bioinformatics analysis resulted in the detection of 24 additional scaffolds,

373 which covered only parts of the astroviral genome (Supplementary Table 2).

374 One scaffold that was identified in a sheep sample (S3.1) had its best hit on the ORF2 region of

375 BoAstV-CH13 (Supplementary Table 2) and clustered near the BoAstV-CH13/NeuroS1 cluster

376 in the phylogenetic tree. Attempts to confirm the sequence of this scaffold by RT-PCR, cloning

377 and further sequencing approaches remained unsuccessful. This may be due to the low viral load

378 or PCR inhibitors in small ruminants' feces that interfere with amplification. The importance of 
379 this finding is so far unclear. With an identity of $57 \%$ on the amino acid level to BoAstV-CH13,

380 this putative astrovirus would be part of the so-called human-mink-ovine-like (HMO) clade,

381 which also comprises the fecal Ovine astrovirus 1 (OvAstV-1) strain as well as the vast majority

382 of encephalitis-associated strains in humans and animals (Kapoor et al. 2009; Reuter et al. 2018).

383 Given the fact that only a part of the sequence (967nt) could be determined, it is still possible

384 that this virus shows varying identities depending on the genome section compared to other viral

385 sequences. However, the importance of a potential occurrence of a novel and divergent

386 neurotropic astrovirus in this fecal sample could not be definitively elucidated.

387 For the remaining 23 scaffolds, best hits were consistently to bovine strains and, unexpectedly,

388 not to previously described ovine (OvAstV-1 and OvAstV-2) (Reuter et al. 2012; Snodgrass \&

389 Gray 1977) and deer astrovirus strains (Smits et al. 2010), respectively. Indeed, sequence

390 similarities compared to best hits in the database entries on protein-level were between $39.3 \%$

391 and $96.4 \%$ (median 72.7\%). 15 scaffolds covered at least a part of the ORF2 and therefore

392 underwent phylogenetic analysis based on the capsid protein precursor sequence together with

393 the 5 newly identified full-length or almost full-length astrovirus genomes (Figure 2). This

394 analysis clearly points out the broad diversity of putative astroviruses detected in this study.

395 Novel sequences were not only discovered in sheep and goat, but also in deer (D2.1, D5.1),

396 interestingly showing a low similarity to known astroviruses in deer (CcAstV-1/DNK/2010,

397 CcAstV-2/DNK/2010). In addition, several sequences found in sheep show a clearly higher

398 identity to bovine astroviruses than to ovine astroviruses (e.g. S6.3* to

399 BoAstV/JPN/Hokkaido12-25/2009). Taken together these results clearly suggest a much larger

400 diversity of astroviruses in small ruminants and deer than known until to date. Moreover, the 
401 occurrence of multiple phylogenetically different astrovirus-like scaffolds in the same sample in

402 most of these animals (e.g. in G1, G8 and S6) supports that astrovirus coinfections are frequent.

403 Five novel, including four full-length and one almost full-length astrovirus sequences were

404 discovered: two in sheep and three in goats. Noteworthy, this study reports for the first time an

405 astrovirus infection in goats. Based on the genetic distances of the capsid protein precursor

406 sequences to the closest related strains, these caprine and ovine astroviruses are grouped into

407 three different genotype species. According to the International Committee on Taxonomy of

408 Viruses ICTV 9th report 2011 (Bosch 2011), amino acid differences between astrovirus

409 genotype species are $>0.338$. Based on this criterion, the strains described in this study belong to

410 three astrovirus genotype species: (i) CapAstV-G2.1 together with BoAstV/JPN/Hokkaido 11-

$41155 / 2009$, (ii) CapAstV-G3.1 on its own and (iii) CapAstV-G5.1 together with OvAstV-S5.1 as

412 well as OvAstV-S6.1. While CapAstV-G2.1 belongs to the same genotype species as

413 BoAstV/JPN/Hokkaido 11-55/2009, CapAstV-G3.1 is sufficiently diverse to build a new

414 genotype species on its own. In particular, CapAstV-G5.1 and OvAstV-S6.1 were very similar to

415 each other, not only in the capsid protein sequence (97.3\%), but also in the predicted non-

416 structural proteins (90.3\%) and on nucleotide level (87.6\%) (Supplementary Table 3). The high

417 similarity between these viruses raises the question of transmission events between sheep and

418 goats and is reminiscent of the situation observed for BoAstV-CH15 and OvAstV-CH16 in

419 astrovirus-associated encephalitis (Boujon et al. 2017b).

420 A retrospective screening of the whole sample panel targeting the five newly discovered

421 astrovirus candidates resulted in only one additional positive sample for target G2.1. This sample

422 originated from the same holding as animal G2 and was collected at the same day. While this

423 screening indicated an overall low prevalence of the newly discovered astrovirus candidates, it 
424 appears plausible that these viruses transmit between animals and that the in herd prevalence of

425 may be much higher.

426 Because recombination between viruses requires infection of the same host cell, the existence of

427 interspecies transmission events are further supported by predicted recombination events

428 (Wolfaardt et al. 2011). In the present study, recombination events were forecast between ovine

429 and caprine strains as well as between caprine and bovine strains. Not only was recombination

430 predicted for OvAstV-S6.1, OvAstV-S5.1 and CapAstV-G5.1, but also for CapAstV-G2.1 and

431 two divergent bovine astroviruses (BoAstV/JPN/Hokkaido11-55/2009, BAstV-

$432 \mathrm{GX} 27 / \mathrm{CHN} / 2014)$, suggesting that these viruses shared the same host at some time point during

433 evolution (Figure 3 and Supplementary Table 4). Similar recombination events have been

434 proposed between a porcine astrovirus and HAstV-3 (Ulloa \& Gutierrez 2010). In addition, the

435 transmission of fecal AstV between cattle and roe deer has been suggested (Smits et al. 2010;

436 Tse et al. 2011). Taken together, all these data challenge the assumption that mammalian

437 astroviruses are strictly host specific. The host-specificity of Astroviridae was already challenged

438 in previous studies (Chu et al. 2010; Karlsson et al. 2015; Rivera et al. 2010).

\section{Conclusion}

440 The question whether enterotropic astroviruses can cause disease in small ruminants remains so

441 far unresolved. Due to the mainly unknown health status of the tested animals, the importance of

442 astroviruses occurring in small ruminants' feces remains so far unclear and needs to be further

443 investigated. Still, this study describes five novel astroviruses discovered in small ruminants,

444 including the first description of an astrovirus in goats and gives new insights into the frequency

445 and diversity of astroviruses in ruminant species. 
447 The authors thank the Institute of Parasitology Vetsuisse Faculty, University of Bern (Bern,

448 Switzerland), especially W. Basso, for providing the fecal samples and S. Schenk as well as M.

449 Fragnière of the Next Generation Sequencing Platform of the University of Bern for performing

450 the high-throughput sequencing experiments.

\section{References}

452

453

454

455

456

457

458

459

460

461

462

463

464

465

466

467

468

469

470

471

472

473

474

475

476

477

478

479

480

481

482

483

484

Appleton H, and Higgins PG. 1975. Letter: Viruses and gastroenteritis in infants. Lancet 1:1297.

Babkin IV, Tikunov AY, Zhirakovskaia EV, Netesov SV, and Tikunova NV. 2012. High evolutionary rate of human astrovirus. Infect Genet Evol 12:435-442. 10.1016/j.meegid.2012.01.019

Blomstrom AL, Widen F, Hammer AS, Belak S, and Berg M. 2010. Detection of a novel astrovirus in brain tissue of mink suffering from shaking mink syndrome by use of viral metagenomics. J Clin Microbiol 48:4392-4396. 10.1128/jcm.01040-10

Bosch A, Guix, S., Krishna, N.K., Méndez, E., Monroe, S.S., Pantin-Jackwood, M. and Schultz-Cherry, S. 2011. ICTV 9th Report (2011): Astroviridae. Available at https://talk.ictvonline.org/ictvreports/ictv 9th report/positive-sense-rna-viruses2011/w/posrna viruses/247/astroviridae2011).

Boujon CL, Koch MC, and Seuberlich T. 2017a. The Expanding Field of Mammalian Astroviruses: Opportunities and Challenges in Clinical Virology. Adv Virus Res 99:109-137. 10.1016/bs.aivir.2017.07.002

Boujon CL, Koch MC, Wuthrich D, Werder S, Jakupovic D, Bruggmann R, and Seuberlich T. 2017b. Indication of Cross-Species Transmission of Astrovirus Associated with Encephalitis in Sheep and Cattle. Emerg Infect Dis 23:1604-1608. 10.3201/eid2309.170168

Bouzalas IG, Wuthrich D, Selimovic-Hamza S, Drogemuller C, Bruggmann R, and Seuberlich T. 2016. Fullgenome based molecular characterization of encephalitis-associated bovine astroviruses. Infect Genet Evol 44:162-168. 10.1016/j.meegid.2016.06.052

Bouzalas IG, Wuthrich D, Walland J, Drogemuller C, Zurbriggen A, Vandevelde M, Oevermann A, Bruggmann R, and Seuberlich T. 2014. Neurotropic astrovirus in cattle with nonsuppurative encephalitis in Europe. J Clin Microbiol 52:3318-3324. 10.1128/JCM.01195-14

Bridger JC. 1980. Detection by electron microscopy of caliciviruses, astroviruses and rotavirus-like particles in the faeces of piglets with diarrhoea. Vet Rec 107:532-533.

Brown JR, Morfopoulou S, Hubb J, Emmett WA, Ip W, Shah D, Brooks T, Paine SM, Anderson G, Virasami A, Tong CY, Clark DA, Plagnol V, Jacques TS, Qasim W, Hubank M, and Breuer J. 2015. Astrovirus VA1/HMO-C: an increasingly recognized neurotropic pathogen in immunocompromised patients. Clin Infect Dis 60:881-888. 10.1093/cid/ciu940

Caul EO, and Appleton H. 1982. The electron microscopical and physical characteristics of small round human fecal viruses: an interim scheme for classification. J Med Virol 9:257-265.

Chu DK, Chin AW, Smith GJ, Chan KH, Guan Y, Peiris JS, and Poon LL. 2010. Detection of novel astroviruses in urban brown rats and previously known astroviruses in humans. J Gen Virol 91:2457-2462. 10.1099/vir.0.022764-0 
485

486

487

488

489

490

491

492

493

494

495

496

497

498

499

500

501

502

503

504

505

506

507

508

509

510

511

512

513

514

515

516

517

518

519

520

521

522

523

524

525

526

527

528

529

530

531

532

Chu DK, Poon LL, Guan Y, and Peiris JS. 2008. Novel astroviruses in insectivorous bats. J Virol 82:91079114. 10.1128/jvi.00857-08

Cordey S, Vu DL, Schibler M, L'Huillier AG, Brito F, Docquier M, Posfay-Barbe KM, Petty TJ, Turin L, Zdobnov EM, and Kaiser L. 2016. Astrovirus MLB2, a New Gastroenteric Virus Associated with Meningitis and Disseminated Infection. Emerg Infect Dis 22:846-853. 10.3201/eid2205.151807

De Battisti C, Salviato A, Jonassen CM, Toffan A, Capua I, and Cattoli G. 2012. Genetic characterization of astroviruses detected in guinea fowl (Numida meleagris) reveals a distinct genotype and suggests cross-species transmission between turkey and guinea fowl. Arch Virol 157:1329-1337. 10.1007/s00705-012-1311-1

De Benedictis P, Schultz-Cherry S, Burnham A, and Cattoli G. 2011. Astrovirus infections in humans and animals - molecular biology, genetic diversity, and interspecies transmissions. Infect Genet Evol 11:1529-1544. 10.1016/j.meegid.2011.07.024

Donato C, and Vijaykrishna D. 2017. The Broad Host Range and Genetic Diversity of Mammalian and Avian Astroviruses. Viruses 9. 10.3390/v9050102

Duffy S, Shackelton LA, and Holmes EC. 2008. Rates of evolutionary change in viruses: patterns and determinants. Nat Rev Genet 9:267-276. 10.1038/nrg2323

Fischer K, Pinho Dos Reis V, and Balkema-Buschmann A. 2017. Bat Astroviruses: Towards Understanding the Transmission Dynamics of a Neglected Virus Family. Viruses 9. 10.3390/v9020034

Gibbs MJ, Armstrong JS, and Gibbs AJ. 2000. Sister-scanning: a Monte Carlo procedure for assessing signals in recombinant sequences. Bioinformatics 16:573-582.

Guan TP, Teng JLL, Yeong KY, You ZQ, Liu H, Wong SSY, Lau SKP, and Woo PCY. 2018. Metagenomic analysis of Sichuan takin fecal sample viromes reveals novel enterovirus and astrovirus. Virology 521:77-91. 10.1016/j.virol.2018.05.027

Hoshino Y, Zimmer JF, Moise NS, and Scott FW. 1981. Detection of astroviruses in feces of a cat with diarrhea. Brief report. Arch Virol 70:373-376.

Kapoor A, Li L, Victoria J, Oderinde B, Mason C, Pandey P, Zaidi SZ, and Delwart E. 2009. Multiple novel astrovirus species in human stool. J Gen Virol 90:2965-2972. 10.1099/vir.0.014449-0

Karlsson EA, Small CT, Freiden P, Feeroz MM, Matsen FAt, San S, Hasan MK, Wang D, Jones-Engel L, and Schultz-Cherry S. 2015. Non-Human Primates Harbor Diverse Mammalian and Avian Astroviruses Including Those Associated with Human Infections. PLoS Pathog 11:e1005225. 10.1371/journal.ppat.1005225

Kjeldsberg E, and Hem A. 1985. Detection of astroviruses in gut contents of nude and normal mice. Brief report. Arch Virol 84:135-140.

Kuchler L, Koch MC, Seuberlich T, and Boujon CL. 2019. Archive Mining Brings to Light a 25-Year Old Astrovirus Encephalitis Case in a Sheep. Front Vet Sci 6:51. 10.3389/fvets.2019.00051

Lam HM, Ratmann O, and Boni MF. 2018. Improved Algorithmic Complexity for the 3SEQ Recombination Detection Algorithm. Mol Biol Evol 35:247-251. 10.1093/molbev/msx263

Lan D, Ji W, Shan T, Cui L, Yang Z, Yuan C, and Hua X. 2011. Molecular characterization of a porcine astrovirus strain in China. Arch Virol 156:1869-1875. 10.1007/s00705-011-1050-8

Laurin MA, Dastor M, and L'Homme Y. 2011. Detection and genetic characterization of a novel pig astrovirus: relationship to other astroviruses. Arch Virol 156:2095-2099. 10.1007/s00705-0111088-7

Le SQ, and Gascuel O. 2008. An improved general amino acid replacement matrix. Mol Biol Evol 25:13071320. 10.1093/molbev/msn067

Li L, Diab S, McGraw S, Barr B, Traslavina R, Higgins R, Talbot T, Blanchard P, Rimoldi G, Fahsbender E, Page B, Phan TG, Wang C, Deng X, Pesavento P, and Delwart E. 2013. Divergent astrovirus associated with neurologic disease in cattle. Emerg Infect Dis 19:1385-1392. 10.3201/eid1909.130682

PeerJ reviewing PDF | (2019:02:34971:1:1:NEW 1 Jun 2019) 
533

534

535

536

537

538

539

540

541

542

543

544

545

546

547

548

549

550

551

552

553

554

555

556

557

558

559

560

561

562

563

564

565

566

567

568

569

570

571

572

573

574

575

576

577

578

579

580

Lum SH, Turner A, Guiver M, Bonney D, Martland T, Davies E, Newbould M, Brown J, Morfopoulou S, Breuer J, and Wynn R. 2016. An emerging opportunistic infection: fatal astrovirus (VA1/HMO-C) encephalitis in a pediatric stem cell transplant recipient. Transpl Infect Dis 18:960-964. 10.1111/tid.12607

Lüthi R, Boujon CL, Kauer R, Koch MC, Bouzalas IG, and Seuberlich T. 2018. Accurate and precise realtime RT-PCR assays for the identification of astrovirus associated encephalitis in cattle. Scientific Reports 8:9215. 10.1038/s41598-018-27533-8

Madeley CR, and Cosgrove BP. 1975. Letter: $28 \mathrm{~nm}$ particles in faeces in infantile gastroenteritis. Lancet 2:451-452.

Marczinke B, Bloys AJ, Brown TD, Willcocks MM, Carter MJ, and Brierley I. 1994. The human astrovirus RNA-dependent RNA polymerase coding region is expressed by ribosomal frameshifting. $J$ Virol 68:5588-5595.

Martin D, and Rybicki E. 2000. RDP: detection of recombination amongst aligned sequences. Bioinformatics 16:562-563.

Martin DP, Murrell B, Golden M, Khoosal A, and Muhire B. 2015. RDP4: Detection and analysis of recombination patterns in virus genomes. Virus Evolution 1:vev003. 10.1093/ve/vev003

Martin DP, Posada D, Crandall KA, and Williamson C. 2005. A modified bootscan algorithm for automated identification of recombinant sequences and recombination breakpoints. AIDS Res Hum Retroviruses 21:98-102. 10.1089/aid.2005.21.98

Matsui M, Ushijima H, Hachiya M, Kakizawa J, Wen L, Oseto M, Morooka K, and Kurtz JB. 1998. Determination of serotypes of astroviruses by reverse transcription-polymerase chain reaction and homologies of the types by the sequencing of Japanese isolates. Microbiol Immunol 42:539547.

Moser LA, and Schultz-Cherry S. 2005. Pathogenesis of astrovirus infection. Viral Immunol 18:4-10. 10.1089/vim.2005.18.4

Nagai M, Omatsu T, Aoki H, Otomaru K, Uto T, Koizumi M, Minami-Fukuda F, Takai H, Murakami T, Masuda T, Yamasato H, Shiokawa M, Tsuchiaka S, Naoi Y, Sano K, Okazaki S, Katayama Y, Oba M, Furuya T, Shirai J, and Mizutani T. 2015. Full genome analysis of bovine astrovirus from fecal samples of cattle in Japan: identification of possible interspecies transmission of bovine astrovirus. Arch Virol 160:2491-2501. 10.1007/s00705-015-2543-7

Oem JK, and An DJ. 2014. Phylogenetic analysis of bovine astrovirus in Korean cattle. Virus Genes 48:372-375. 10.1007/s11262-013-1013-0

Padidam M, Sawyer S, and Fauquet CM. 1999. Possible emergence of new geminiviruses by frequent recombination. Virology 265:218-225. 10.1006/viro.1999.0056

Pfaff F, Schlottau K, Scholes S, Courtenay A, Hoffmann B, Hoper D, and Beer M. 2017. A novel astrovirus associated with encephalitis and ganglionitis in domestic sheep. 64:677-682. 10.1111/tbed.12623

Posada D, and Crandall KA. 2001. Evaluation of methods for detecting recombination from DNA sequences: computer simulations. Proc Natl Acad Sci U S A 98:13757-13762. 10.1073/pnas. 241370698

Reuter G, Pankovics P, and Boros A. 2018. Nonsuppurative (Aseptic) Meningoencephalomyelitis Associated with Neurovirulent Astrovirus Infections in Humans and Animals. Clin Microbiol Rev 31. 10.1128/cmr.00040-18

Reuter G, Pankovics P, Delwart E, and Boros A. 2012. Identification of a novel astrovirus in domestic sheep in Hungary. Arch Virol 157:323-327. 10.1007/s00705-011-1151-4

Rivera R, Nollens HH, Venn-Watson S, Gulland FM, and Wellehan JF, Jr. 2010. Characterization of phylogenetically diverse astroviruses of marine mammals. J Gen Virol 91:166-173. 10.1099/vir.0.015222-0

Peer) reviewing PDF | (2019:02:34971:1:1:NEW 1 Jun 2019) 
581

582

583

584

585

586

587

588

589

590

591

592

593

594

595

596

597

598

599

600

601

602

603

604

605

606

607

608

609

610

611

612

613
Schlottau K, Schulze C, Bilk S, Hanke D, Hoper D, Beer M, and Hoffmann B. 2016. Detection of a Novel Bovine Astrovirus in a Cow with Encephalitis. Transbound Emerg Dis 63:253-259. 10.1111/tbed.12493

Sebire NJ, Malone M, Shah N, Anderson G, Gaspar HB, and Cubitt WD. 2004. Pathology of astrovirus associated diarrhoea in a paediatric bone marrow transplant recipient. J Clin Pathol 57:10011003. 10.1136/jcp.2004.017178

Seuberlich T, Wuthrich D, Selimovic-Hamza S, Drogemuller C, Oevermann A, Bruggmann R, and Bouzalas I. 2016. Identification of a second encephalitis-associated astrovirus in cattle. Emerg Microbes Infect 5:e71. 10.1038/emi.2017.56

Smith JM. 1992. Analyzing the mosaic structure of genes. Journal of Molecular Evolution 34:126-129. 10.1007/BF00182389

Smits SL, van Leeuwen M, Kuiken T, Hammer AS, Simon JH, and Osterhaus AD. 2010. Identification and characterization of deer astroviruses. J Gen Virol 91:2719-2722. 10.1099/vir.0.024067-0

Snodgrass DR, and Gray EW. 1977. Detection and transmission of $30 \mathrm{~nm}$ virus particles (astroviruses) in faeces of lambs with diarrhoea. Arch Virol 55:287-291.

Tse H, Chan WM, Tsoi HW, Fan RY, Lau CC, Lau SK, Woo PC, and Yuen KY. 2011. Rediscovery and genomic characterization of bovine astroviruses. J Gen Virol 92:1888-1898. 10.1099/vir.0.030817-0

Tzipori S, Menzies JD, and Gray EW. 1981. Detection of astrovirus in the faeces of red deer. Vet Rec 108:286.

Ulloa JC, and Gutierrez MF. 2010. Genomic analysis of two ORF2 segments of new porcine astrovirus isolates and their close relationship with human astroviruses. Can J Microbiol 56:569-577. 10.1139/w10-042

Willcocks MM, Ashton N, Kurtz JB, Cubitt WD, and Carter MJ. 1994. Cell culture adaptation of astrovirus involves a deletion. J Virol 68:6057-6058.

Williams FP, Jr. 1980. Astrovirus-like, coronavirus-like, and parvovirus-like particles detected in the diarrheal stools of beagle pups. Arch Virol 66:215-226.

Wolfaardt M, Kiulia NM, Mwenda JM, and Taylor MB. 2011. Evidence of a recombinant wild-type human astrovirus strain from a Kenyan child with gastroenteritis. J Clin Microbiol 49:728-731. 10.1128/jcm.01093-10

Woode GN, and Bridger JC. 1978. Isolation of small viruses resembling astroviruses and caliciviruses from acute enteritis of calves. J Med Microbiol 11:441-452. 10.1099/00222615-11-4-441 


\section{Table $\mathbf{1}$ (on next page)}

Discovery of five novel full-length astrovirus genomes in sheep and goats.

Best hits of the bioinformatics pipeline on nucleotide (full-genome) and amino acid (ORF lab, ORF2) level are presented separately. Genbank accession number are provided in brackets. BoAstV, bovine astrovirus; CapAstV, caprine astrovirus; OvAstV, ovine astrovirus. 


\begin{tabular}{|c|c|c|c|c|c|c|}
\hline & $\begin{array}{l}\text { Full genome best hit } \\
\text { (fullgenome-accession) }\end{array}$ & $\begin{array}{c}\text { Full } \\
\text { genome } \\
\text { identity } \\
{[\%]}\end{array}$ & $\begin{array}{c}\text { ORF 1ab best hit } \\
\text { non-structural protein } \\
\text { (1ab-BLASTP-accession) }\end{array}$ & $\begin{array}{c}\text { ORF1ab } \\
\text { identity [\%] } \\
\text { non- } \\
\text { stuctural } \\
\text { proteins } \\
\end{array}$ & $\begin{array}{c}\text { ORF2 best hit } \\
\text { capsid protein } \\
\text { (2-BLASTP-accession) }\end{array}$ & $\begin{array}{c}\text { ORF2 } \\
\text { Identity } \\
{[\%]} \\
\text { capsid } \\
\text { protein }\end{array}$ \\
\hline $\begin{array}{c}\text { CapAstV- } \\
\text { G2.1 } \\
(\mathrm{MK} 404645.1)\end{array}$ & $\begin{array}{c}\text { Bovine astrovirus } \\
\text { JPN/Hokkaido11-55/2009 } \\
\text { (LC047790.1) }\end{array}$ & 76.6 & $\begin{array}{c}\text { BoAstV/JPN/Hokkaido11- } \\
55 / 2009 \\
\text { (BAS29607.1) }\end{array}$ & 75.4 & $\begin{array}{c}\text { BoAstV/JPN/Hokkaido11- } \\
55 / 2009 \\
\text { (BAS29609.1) }\end{array}$ & 73.7 \\
\hline $\begin{array}{c}\text { CapAstV- } \\
\text { G3.1 } \\
(\mathrm{MK} 404646.1) \\
\end{array}$ & $\begin{array}{c}\text { Bovine astrovirus B170/HK } \\
\text { (HQ916314.1) }\end{array}$ & 69 & $\begin{array}{l}\text { Sichuan takin astrovirus } \\
\quad\left(Y P_{0} \text { 009480536.1) }\right.\end{array}$ & 71.7 & $\begin{array}{l}\text { Bovine astrovirus B170/HK } \\
\text { (YP_009010954.1) }\end{array}$ & 63.9 \\
\hline $\begin{array}{c}\text { CapAstV- } \\
\text { G5.1 } \\
(\mathrm{MK} 404647.1)\end{array}$ & $\begin{array}{l}\text { Sichuan takin astrovirus } \\
\text { (NC_037655.1) }\end{array}$ & 70.9 & $\begin{array}{l}\text { Bovine astrovirus B76-2/HK } \\
\text { (YP_009010946.1) }\end{array}$ & 75.1 & $\begin{array}{c}\text { Astrovirus deer/CcAstV- } \\
\text { 1/DNK/2010 } \\
\text { (ADO67579.1) }\end{array}$ & 57.7 \\
\hline $\begin{array}{c}\text { OvAstV-S5.1 } \\
(\text { MK404648.1) }\end{array}$ & $\begin{array}{c}\text { Bovine astrovirus B76-2/HK } \\
\text { (HQ916317.1) }\end{array}$ & 70.5 & $\begin{array}{l}\text { Bovine astrovirus B76-2/HK } \\
\text { (YP_009010946.1) }\end{array}$ & 73.8 & $\begin{array}{c}\text { Astrovirus deer/CcAstV- } \\
\text { 1/DNK/2010 } \\
\text { (ADO67579.1) }\end{array}$ & 59.0 \\
\hline $\begin{array}{c}\text { OvAstV-S6.1 } \\
(\text { MK404649.1) }\end{array}$ & $\begin{array}{l}\text { Sichuan takin astrovirus } \\
\quad(\text { NC_037655.1) }\end{array}$ & 70.8 & $\begin{array}{c}\text { BoAstV/JPN/Ishikawa24-6/2013 } \\
\text { (BAS29598.1) }\end{array}$ & 74.6 & $\begin{array}{c}\text { Astrovirus deer/CcAstV- } \\
\text { 1/DNK/2010 } \\
\text { (ADO67579.1) }\end{array}$ & 57.5 \\
\hline
\end{tabular}


Figure 1 (on next page)

Virus discovery workflow in small ruminants' feces samples. 
PCR-screening

Fecal sample resuspension

RNA extraction

qRT-PCR for neurotropic AstV

\& PanAstV-RT-PCR

Selection of PCR-positive samples for NGS
PeerJ Pre-treatment for ${ }^{N}$ NGS to be revie

\section{Bioinformatics pipeline}

\begin{tabular}{|c|}
\hline Selected samples \\
\hline Resuspension 1:5 in PBS \\
\hline Centrifugation \& Filtration \\
\hline Benzonase-digestion \\
\hline RNA extraction \\
\hline NGS
\end{tabular}

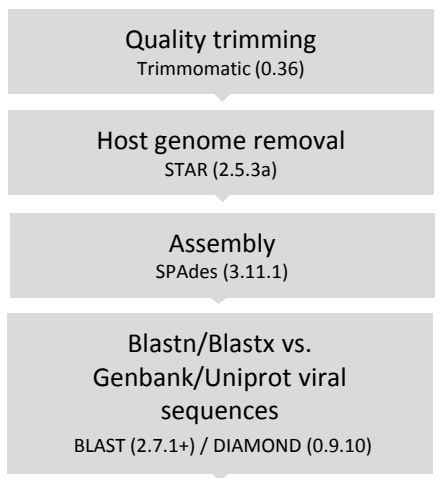

Selection of scaffolds with AstV hits 


\section{Figure 2 (on next page)}

Phylogenetic analysis of novel small ruminant astroviruses.

Phylogenetic analysis using the Maximum Likelihood method, based on 76 amino acid sequences of the capsid precursor protein of selected astrovirus strains together with the ones generated by the bioinformatics pipeline, with the sheep (blue), goat (green), and deer sequences (purple) from this study marked. GenBank accession numbers are provided in brackets. Filled rhombi indicate encephalitis-associated strains described in animals. Capsid protein precursor sequences translated from scaffolds with less than three identified ORFs are marked with an asterisk. AvAstV, avian astrovirus; BoAstV, bovine astrovirus; CcAstV, deer astrovirus; DromAstV, dromedary astrovirus; HuAstV, human astrovirus; MiAstV, mink astrovirus; PoAstV, porcine astrovirus; StAstV, sichuan takin astrovirus; WBufAstV, water buffalo astrovirus; YakAstV, yak astrovirus. 
64 ـ CcAstV-2/DNK/2010 (ADO67581.1)

$22 \square$ BoAstV-B18/HK (AED89600.1)

28 BoAstV-GX7/CHN/2014 (YP 009041464.1)

9. 9 StAstV (YP 009480538.1)

92

CcAstV-1/DNK/2010 (ADO67579.1)

86

99 G1.3*

$\mathrm{G} 2.2^{*}$

\section{PeerJ}

42

35

95 DromAstV (YP 009162619.1)

51

${ }_{99}{ }_{95}{ }_{\text {OvAstV-S6.1 (MK404649.1) }}^{\text {CapAstV-G5.1 (MK404647.1) }}$

(Y) 009010945.1)

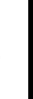

W9 WBufAstV/ITA/2013/750(ALT13998.1)

42

52

48

BoAstV/ITA/2012/715 (ALT14000.1)

$48 \lim _{41}$ WBufAstV/ITA/2013/619 (ALT13996.1)

99

${ }^{\mathrm{G} 8.1^{*}}$

- BoAstV/JPN/Kagoshima2-3-2/2015 (BAS29633.1)

42 CapAstV-G2.1 (MK404645.1)

90 G3.2*

73 BoAstV/JPN/Hokkaido11-55/2009 (BAS29609.1)

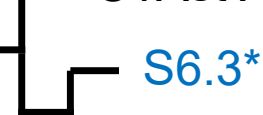

${ }_{93}$ BoAstV/JPN/Hokkaido12-25/2009 (BAS29618.1)

HuAstV-1 (NP 059444.1)

52 LuAstV-MLB1 (ACI62175.1)

$\bigsqcup_{87}[$ HuAstV-MLB2 (AER41414.1)

99

PoAstV-3/NI/HUN

PoAstV-3/US-MO123 (AFW16977.1)

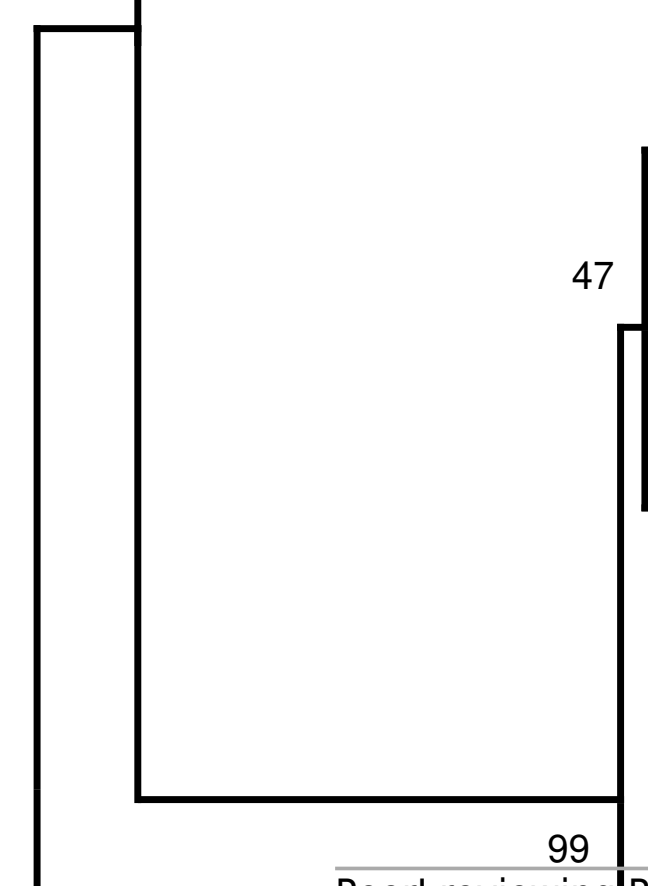

47

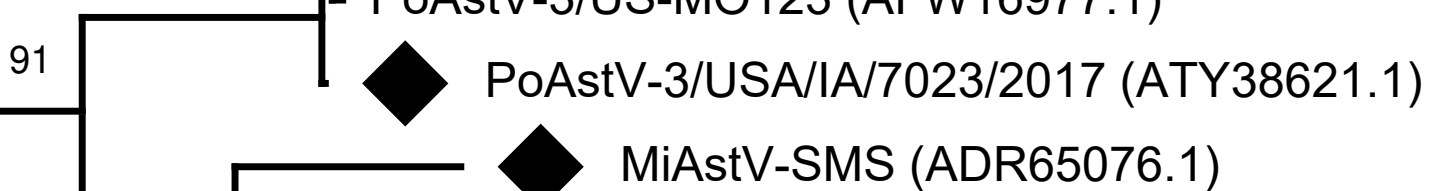

99 MiAstV (NP 795336.1)

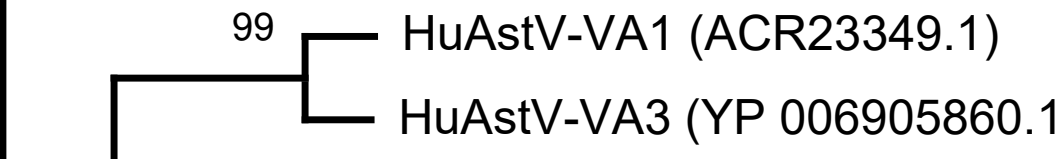

93

HuAstV-VA5 (AJI44022.1)

— HuAstV-VA2 (YP 003275953.1)

$99-$ HuAstV-VA4 (AFV53441.1)

ing

70

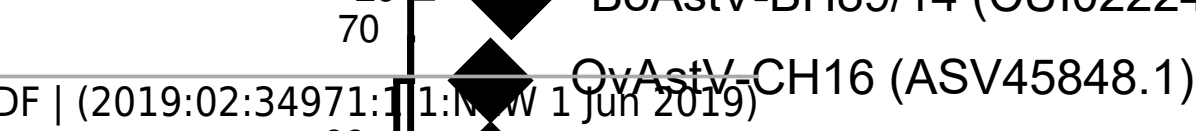

9 BoAstV-CH15 (ALQ33326.2)

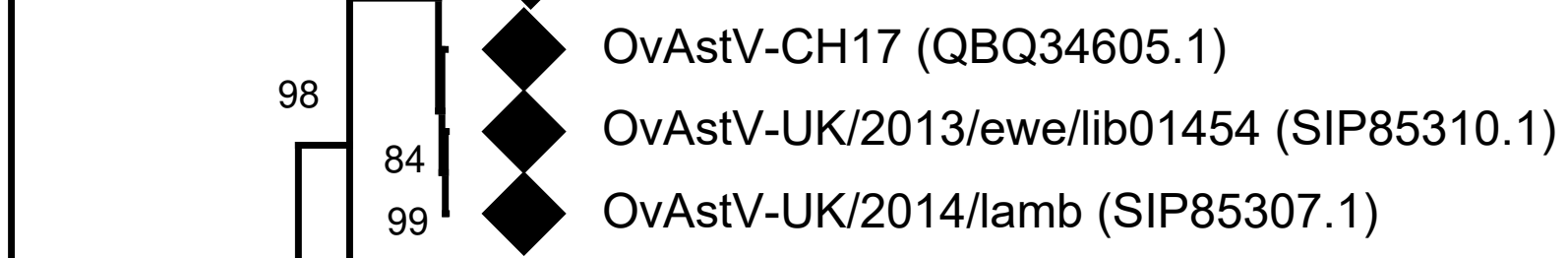

99

- OvAstV-1 (NP 059946.1)

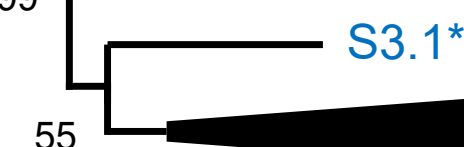

99

BoAstV-CH13/NeuroS1

AvAstV (ADG45753.1)

genogroup I 


\section{Figure 3}

Recombination analysis of newly discovered full-length astrovirus genomes.

(A) Predicted recombination events 1 and 3 between CapAstV-G5.1, OvAstV-S5.1 and OvAstV-S6.1, where all three sequences may be the resulting recombinant. (B) Predicted recombination event 2 with CapAstV-G2.1 as the recombinant, BoAstV/GX27 as the minor parent and BoAstV/JPN/HK as the major parent. Plots were constructed using the RDP Method graphical output in RDP4. Nucleotide positions within the astrovirus genome are depicted on the axis of abscissas in $\mathrm{kb}$. Red bars schematically indicate the parts of the genomes involved in the recombination events. For comparison, the astrovirus genome organization is presented at the bottom. BoAstV/JPN/HK, Bovine astrovirus genomic RNA, nearly complete genome, strain: BoAstV/JPN/Hokkaido11-55/2009 (LC047790.1) BoAstV/GX27, Bovine astrovirus strain BAstV-GX27/CHN/2014, complete genome (KJ620980.1) CapAstV, Caprine Astrovirus OvAstV, Ovine Astrovirus 


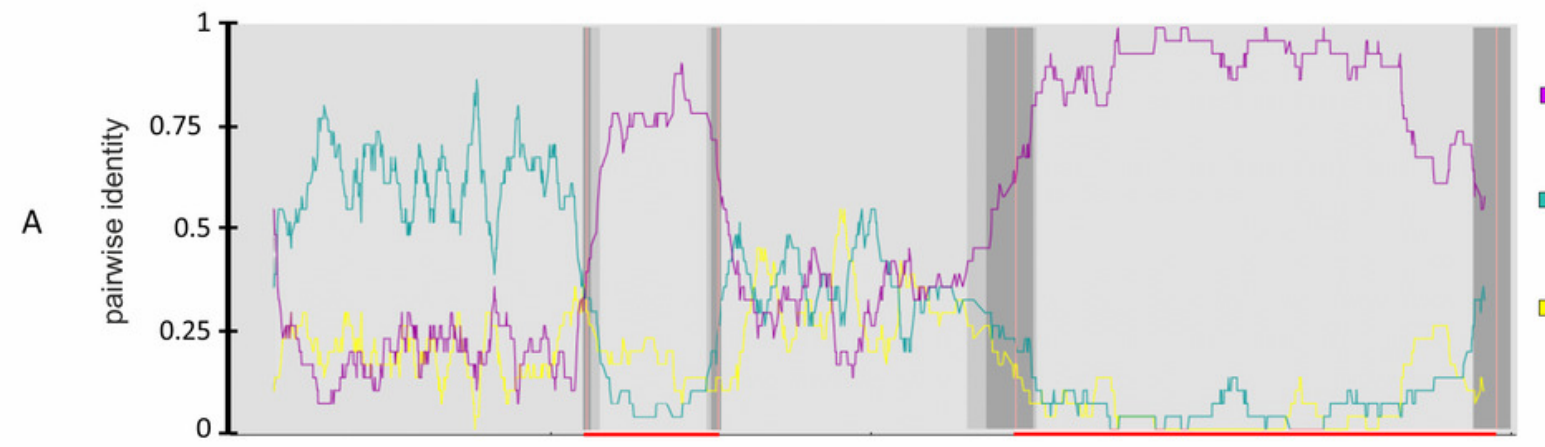

- CapAstV/G5.1 - OvAstV/S6.1

OvAstV/S5.1OvAstV/S6.1

$\square \quad$ OvAstV/S5.1 CapAstV/G5.1
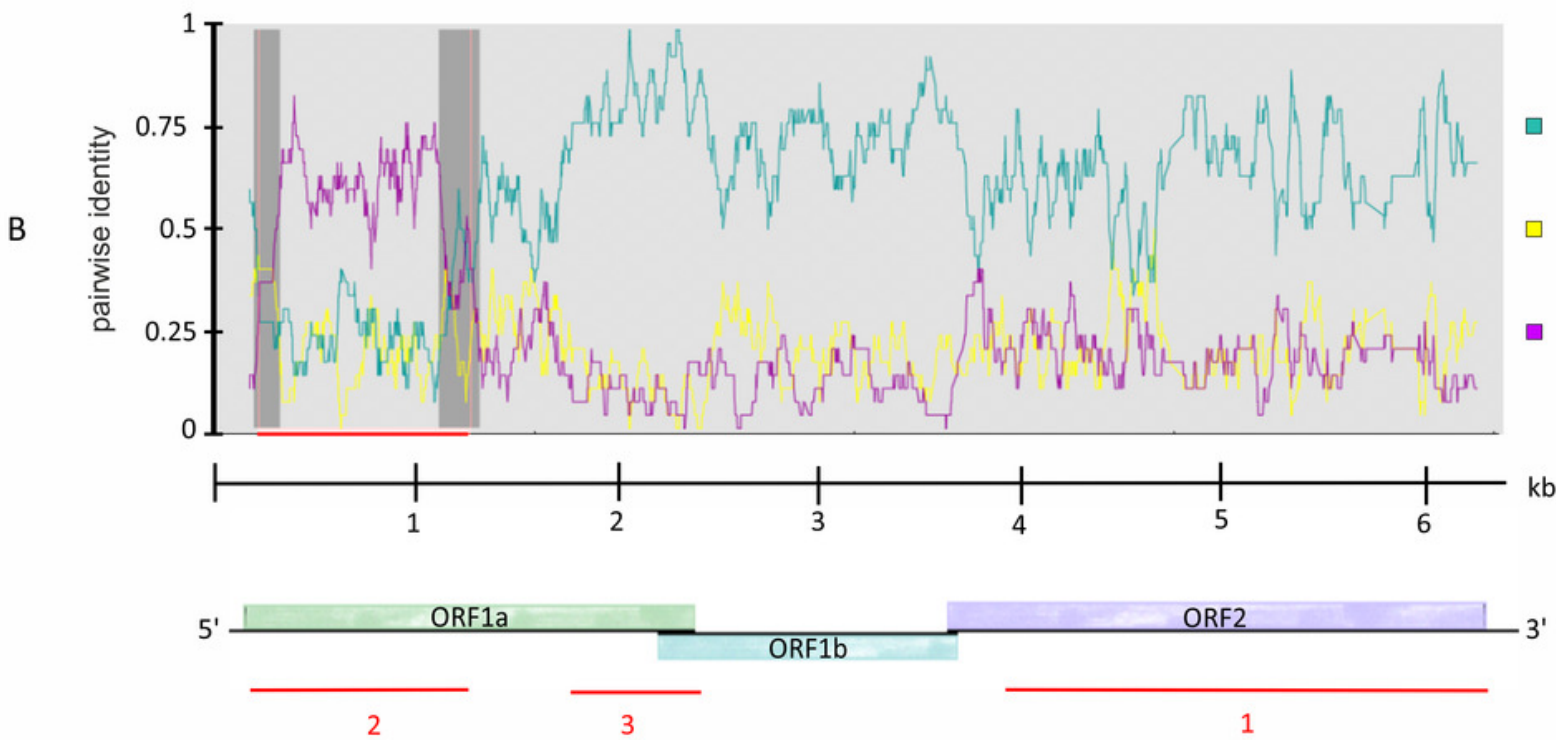

BoAstV/JPN/HK CapAstV/G2.1

BoAstV/JPN/HK BoAstV/GX27

BoAstV/GX27 CapAstV/G2.1 